\title{
L-Fuzzy Filters of a Poset
}

\author{
Berhanu Assaye Alaba, Mihret Alamneh and Derso Abeje
}

\begin{abstract}
Many generalizations of ideals and filters of a lattice to an arbitrary poset have been studied by different scholars. The authors of this paper introduced several generalizations of $L$-fuzzy ideal of a lattice to an arbitrary poset in [1]. In this paper, we introduce several $L$-fuzzy filters of a poset which generalize the $L$-fuzzy filter of a lattice and give several characterizations of them.

Index Terms-Poset, Filter, L-fuzzy closed filter, L-fuzzy Frink filter, L-fuzzy V-Filter, L-fuzzy semi-filter, L-fuzzy filter, l-L-fuzzy filter.
\end{abstract}

\section{INTRODUCTION}

W E have found several generalizations of ideals and filters of a lattice to arbitrary poset (partially ordered set) in a literature. Birkhoff in [2, p. 59] introduced a closed or normal ideals who gives accredit to the work of Stone in [3]. Next, in 1954 the second type of ideal and filter of a poset called Frink ideal and Frink filter have been introduced by O. Frink [4]. Following this P. V. Venkatanarasimhan developed the theory of semi ideals and semi filter in [5] and ideals and filters for a poset in [6], in 1970. These ideals (respectively, filters) are called ideals (respectively, filter) in the sense of Venkatanarasimhan or V-ideals (V-filters) for short. Later Halaś [7], in 1994, introduced a new ideal and filter of a poset which seems to be a suitable generalization of the usual concept of ideal and filter in a lattice. We will simply call it ideal (respectively, filter) in the sense of Halaš.

Moreover, the concept of fuzzy ideals and filters of a lattice has been studied by different authors in series of papers [8], [9], [10], [11] and [12]. The aim of this paper is to notify several generalizations of $L$-fuzzy filters of a lattice to an arbitrary poset whose truth values are in a complete lattice satisfying the infinite meet distributive law and give several characterizations of them. We also prove that the set of all $L$-fuzzy filters of a poset forms a complete lattice with respect to point-wise ordering " $\subseteq$ ". Throughout this work, $L$ means a non-trivial complete lattice satisfying the infinite meet distributive law: $x \wedge \sup S=\sup \{x \wedge s: s \in S\}$ for all $x \in L$ and for any subset $S$ of $L$.

\section{PRELIMINARIES}

We briefly recall certain necessary concepts, terminologies and notations from [2], [13] and [14]. A binary relation $" \leq "$ on a non-empty set $Q$ is called a partial order if it is reflexive, anti-symmetric and transitive. A pair $(Q, \leq)$ is

Manuscript received June 25, 2018; accepted March 15, 2019.

B.A. Alaba and M. Alamneh are with the College of Science, Bahir Dar University, Bahir Dar, Ethiopia. E-mails: berhanu_assaye@yahoo.com, deab02@yahoo.com

D. Abeje is with the Department of Mathematics, College of Science, University of Gondar, Gondar, Ethiopia. E-mail: mihretmahlet@yahoo. com called a partially ordered set or simply a poset if $Q$ is a nonempty set and " $\leq "$ is a partial order on $Q$. When confusion is unlikely, we use simply the symbol $Q$ to denote a Poset $(Q, \leq)$. Let $Q$ be a poset and $S \subseteq Q$. An element $x$ in $Q$ is called a lower bound (respectively, an upper bound) of $S$ if $x \leq a$ (respectively, $x \geq a$ ) for all $a \in S$. We denote the set of all lower bounds and upper bounds of $S$ by $S^{l}$ and $S^{u}$, respectively. That is $S^{l}=\{x \in Q: x \leq a \forall a \in S\}$ and $S^{u}=\{x \in Q: x \geq a \forall a \in S\}$. $S^{u l}$ shall mean $\left\{S^{u}\right\}^{l}$ and $S^{l u}$ shall mean $\left\{S^{l}\right\}^{u}$. Let $a, b \in Q$. Then $\{a\}^{u}$ is simply denoted by $a^{u}$ and $\{a, b\}^{u}$ is denoted by $(a, b)^{u}$. Similar notations are used for the set of lower bounds. We note that $S \subseteq S^{u l}$ and $S \subseteq S^{l u}$ and if $S \subseteq T$ in $Q$ then $S^{l} \supseteq T^{l}$ and $S^{u} \supseteq T^{u}$. Moreover, $S^{l u l}=S^{l}, S^{u l u}=S^{u},\left\{a^{u}\right\}^{l}=a^{l}$ and $\left\{a^{l}\right\}^{u}=a^{u}$. An element $x_{0}$ in $Q$ is called the least upper bound of $S$ or supremum of $\mathrm{S}$, denoted by supS (respectively, the greatest lower bound of $\mathrm{S}$ or infimum of $\mathrm{S}$, denoted by infS) if $x_{0} \in S^{u}$ and $x_{0} \leq x$ $\forall x \in S^{u}$ (respectively, if $x_{0} \in S^{l}$ and $x \leq x_{0} \forall x \in S^{l}$ ). An element $x_{0}$ in $Q$ is called the largest (respectively, the smallest) element if $x \leq x_{0}$ (respectively, $x_{0} \leq x$ ) for all $x \in Q$. The largest (respectively, the smallest) element if it exists in $Q$ is denoted by 1 (respectively, by 0 ). A poset $(Q \leq)$ is called bounded if it has 0 and 1 . Note that if $S=\emptyset$ we have $S^{l u}=\left(\emptyset^{l}\right)^{u}=Q^{u}$ which is equal to the empty set or the singleton set $\{1\}$ if $Q$ has the largest element 1

Now we recall definitions of filters of a poset that are introduced by different scholars.

Definition 2.1 (Dual of [2]): A subset $F$ of a poset $(Q, \leq)$ is said to be a closed or a normal filter in $Q$ if $F^{l u} \subseteq F$.

Definition 2.2 ([4]): A subset $F$ of a poset $(Q, \leq)$ is said to be a Frink filter in $Q$ if $S^{l u} \subseteq F$ whenever $S$ is a finite subset of $F$.

Definition 2.3 ([5]): A non-empty subset $F$ of a poset $(Q, \leq)$ is called a semi-filter or an order filter of $Q$ if $a \leq b$ and $a \in F$ implies $b \in F$.

Definition 2.4 ([6]): A subset $F$ of a poset $(Q, \leq)$ is said to be a V-filter or a filter in the sense of Venkatannarasimhan if $F$ is a semi-filter and for any nonempty finite subset $S$ of $F$, if inf $S$ exists, then $\inf S \in F$.

Definition 2.5 ([7]): A subset $F$ of a poset $(Q, \leq)$ is called a filter in $Q$ in the sense of Halaš if $(a, b)^{l u}$ contained in $F$ whenever $a, b \in F$.

Note that every filter of a poset $Q$ defined above contains $Q^{u}$.

Remark 2.6: The following remarks are due to R. Halaš and J. Rachŭnek [15].

1) If $(Q \leq)$ is a lattice then a non-empty subset $F$ of $Q$ is a filter as a poset if and only if it is a filter as a lattice $(Q \leq)$.

2) If a poset does not have the largest element then the empty subset $\emptyset$ is a filter in $(Q \leq)$ ( since $\emptyset^{l u}=\left(\emptyset^{l}\right)^{u}=$ $\left.Q^{u}=\emptyset\right)$ 
Definition 2.7: Let $A$ be any subset of a poset $Q$. Then the smallest filter containing $A$ is called a filter generated by $A$ and is denoted by $[A)$. The filter generated by a singleton set $\{a\}$, is called a principal filter and is denoted by $[a)$ Note that for any subset $S$ of $Q$ if inf $S$ exists then $S^{l u}=[\inf S$ ).

The followings are some characterizations of filters generated by a subset $S$ of a poset $Q$. We write $T \subset \subset S$ to mean $T$ is a finite subset of $S$.

1) The closed or normal filter generated by $S$, denoted by $[S)_{C}$, is $[S)_{C}=\bigcup\left\{T^{l u}: T \subseteq S\right\}$ where the union is taken over all subsets $T$ of $S$.

2) The Frink filter generated by $S$, denoted by $[S)_{F}$, is $[S)_{F}=\bigcup\left\{T^{l u}: T \subset \subset S\right\}$, where the union is taken over all finite subsets $T$ of $S$

3) Define $B_{1}=\bigcup\left\{(a, b)^{l u}: a, b \in S\right\}$ and $B_{n}=\bigcup\left\{(a, b)^{l u}\right.$ : $\left.a, b \in B_{n-1}\right\}$ for each positive integer $n \geq 2$, inductively. Then the filter generated by $S$ in the sense of Halaš, denoted by $[S)_{H}$, is $[S)_{H}=\bigcup\left\{B_{n}: n \in \mathscr{N}\right\}$ where $\mathscr{N}$ denotes the set of positive integers.

4) If $a \in Q$ then $[a)=\{x \in Q: x \leq a\}=a^{l}$ is the principal ideal generated by $a$.

Definition 2.8 ([7]): A filter $F$ of a poset $Q$ is called an $l$-filter if $(x, y)^{l} \cap F \neq \emptyset$ for all $x, y \in F$.

Note that an easy induction shows that $F$ is an $l$-filter if $B^{l} \cap$ $F \neq \emptyset$ for every non-empty finite subset $B$ of $F$.

Theorem 2.9 ([7]): Let $\mathscr{F}(Q)$ be the set of filters of a poset $Q$ and $A$ and $B$ be $l$-filters of $Q$. Then the supremum $A \vee B$ of $A$ and $B$ in $\mathscr{F}(Q)$ is $A \vee B=\bigcup\left\{(a, b)^{l u}: a \in A, b \in B\right\}$.

Definition 2.10 ([16]): An $L$-fuzzy subset $\eta$ of a poset $Q$ is a function from $Q$ into $L$.

Note that if $L$ is a unit interval of real numbers [0,1], then the $L$-fuzzy subset $\eta$ is the fuzzy subsets of $Q$ which is introduced by L. Zadeh [17]. The set of all $L$-fuzzy subsets of $Q$ is denoted by $L^{Q}$.

Definition 2.11 ([11]): Let $\eta \in L^{Q}$. Then for each $\alpha \in L$ the set $\eta_{\alpha}=\{x: \eta(x) \geq \alpha\}$ is called the level subset or level cut of $\eta$ at $\alpha$.

Lemma 2.12 ([9]): Let $\eta \in L^{Q}$. Then $\eta(x)=\sup \{\alpha \in L$ : $\left.x \in \eta_{\alpha}\right\}$ for all $x \in Q$.

Definition 2.13 ([16]): Let $v, \sigma \in L^{Q}$. Define a binary relation " $\subseteq$ " on $L^{Q}$ by $v \subseteq \sigma$ if and only $v(x) \leq \sigma(x)$ for all $x \in Q$.

It is simple to verify that the binary relation " $\subseteq$ " on $L^{Q}$ is a partial order and it is called the point wise ordering.

Definition 2.14 ([18]): Let $\theta$ and $\eta$ be in $L^{Q}$. Then the union of fuzzy subsets $\theta$ and $\eta$ of $X$, denoted by $\theta \cup \eta$, is a fuzzy subset of $Q$ defined by $(\theta \cup \eta)(x)=\theta(x) \vee \eta(x)$ for all $x \in Q$ and the intersection of fuzzy subsets $\theta$ and $\eta$ of $Q$, denoted by $\theta \cap \eta$, is a fuzzy subset of $X$ defined by $(\theta \cap \eta)(x)=$ $\theta(x) \wedge \eta(x)$ for all $x \in Q$.

More generally, the union and intersection of any family $\left\{\eta_{i}\right\}_{i \in \Delta}$ of $L$-fuzzy subsets of $Q$, denoted by $\bigcup_{i \in \Delta} \eta_{i}$ and $\bigcap_{i \in \Delta} \eta_{i}$ respectively, are defined by:

$\left(\bigcup_{i \in \Delta} \eta_{i}\right)(x)=\sup _{i \in \Delta} \eta_{i}(x)$ and $\bigcap_{i \in \Delta} \eta_{i}=\inf _{i \in \Delta} \eta_{i}(x)$ for all $x \in Q$, respectively.

Definition 2.15 ([10]): An $L$-fuzzy subset $\eta$ of a lattice $Q$ with 1 is said to be an $L$-fuzzy filter of $Q$; if $\eta(1)=1$ and $\eta(a \wedge b)=\eta(a) \wedge \eta(b)$ for all $a, b \in Q$.
Definition 2.16: Let $\eta$ be $L$ - fuzzy subset of a poset $Q$. The smallest fuzzy filter of $Q$ containing $\eta$ is called a fuzzy filter generated by $\eta$ and is denoted by $[\eta)$.

\section{L-FuZZY Filters OF A POSET}

In this section, we notify the concept of $L$-fuzzy filters of a poset and give several characterizations of them. Throughout this paper, $Q$ stands for a poset $(Q, \leq)$ with 1 unless otherwise stated. We begin with the following

Definition 3.1: An $L$-fuzzy subset $\eta$ of $Q$ is called an $L$ fuzzy closed filter if it fulfills the following conditions:

1) $\eta(1)=1$ and

2) for any subset $S$ of $Q, \eta(x) \geq \inf \{\eta(a): a \in S\} \forall x \in S^{l u}$.

Lemma 3.2: A subset $F$ of $Q$ is a closed filter of $Q$ if and only if its characteristic map $\chi_{F}$ is an $L$-fuzzy closed filter of $Q$.

Proof: Suppose $F$ is a closed filter of $Q$. Since 1 is in $F^{l u} \subseteq F$, we have $\chi_{F}(1)=1$. Again let $S$ be any subset of $\mathrm{Q}$ and $x \in S^{l u}$. Then if $S \subseteq F$, we have $S^{l u} \subseteq F^{l u} \subseteq F$ and $\chi_{F}(a)=1$ for all $a \in S$. Therefore $\chi_{F}(x)=1=\inf \left\{\chi_{F}(a): a \in\right.$ $S\}$. Again if $S \nsubseteq F$, then there is $c \in S$ such that $c \notin F$ and hence $\chi_{F}(c)=0$ and hence $\chi_{F}(x) \geq 0=\inf \left\{\chi_{F}(a): a \in S\right\}$. Thus in either cases, $\chi_{F}(x) \geq \inf \left\{\chi_{F}(a): a \in S\right\}$ for all $x \in$ $S^{l u}$ and $S \subseteq Q$. Therefore, $\chi_{F}$ is an $L$-fuzzy closed filter of $Q$. Conversely, suppose $\chi_{F}$ is an $L$-fuzzy closed filter. Since $\chi_{F}(1)=1$, we have $1 \in F$, that is $\{1\}=Q^{u} \subseteq F$. Let $x \in F^{l u}$. Then by hypotheses, $\chi_{F}(x) \geq \inf \left\{\chi_{F}(a): a \in F\right\}=1$. This implies $\chi_{F}(x)=1$ and hence $x \in F$. Therefore, $F^{l u} \subseteq F$ and hence $F$ is a closed filter. This proves the result.

The following result characterizes the $L$-fuzzy closed filter of $Q$ in terms of its level subsets.

Lemma 3.3: Let $\eta$ be in $L^{Q}$. Then $\eta$ is an $L$ - fuzzy closed filter of $Q$ if and only if $\eta_{\alpha}$ is a closed filter of $Q$ for all $\alpha \in L$.

Proof: Let $\eta$ be an $L$ - fuzzy closed filter of $Q$ and $\alpha \in L$. Then $\eta(1)=1 \geq \alpha$ and hence $1 \in \eta_{\alpha}$, i.e., $\{1\}=Q^{u} \subseteq \eta_{\alpha}$. Again let $x \in\left(\eta_{\alpha}\right)^{l u}$. Then $\eta(x) \geq \inf \left\{\eta(a): a \in \eta_{\alpha}\right\} \geq \bar{\alpha}$ and hence $x \in \eta_{\alpha}$. Therefore $\left(\eta_{\alpha}\right)^{l u} \subseteq \eta_{\alpha}$ and hence $\eta_{\alpha}$ is a closed filter.

Conversely, let $\eta_{\alpha}$ is a closed filter of $Q$ for all $\alpha \in L$. In particular $\eta_{1}$ is a closed filter. Since $1 \in\left(\eta_{1}\right)^{l u} \subseteq \eta_{1}$, we have $\eta(1)=1$.

Again let $S$ be any subset of $Q$. Put $\alpha=\inf \{\eta(a): a \in S\}$. Then $\eta(a) \geq \alpha \forall a \in S$ and hence $S \subseteq \mu_{\alpha}$. This implies $S^{l u} \subseteq$ $\mu_{\alpha}^{l u} \subseteq \mu_{\alpha}$. Now $x \in S^{l u} \Rightarrow x \in \eta_{\alpha} \Rightarrow \eta(x) \geq \alpha=\inf \{\eta(a): a \in$ $S\}$. Therefore $\eta$ is an $L$-fuzzy closed filter of $Q$. This proves the result.

Lemma 3.4: Let $\eta$ be fuzzy closed filter of a poset $Q$. Then $\eta$ is iso-tone, in the sense that $\eta(x) \leq \eta(y)$ whenever $x \leq y$.

Proof: Let $x, y \in Q$ such that $x \leq y$. Put $\eta(x)=\alpha$. Since $\eta$ is a fuzzy closed filter, $\eta_{\alpha}$ is a closed filter of $Q$ and hence $\left(\eta_{\alpha}\right)^{l u} \subseteq \eta_{\alpha}$. Now $\eta(x)=\alpha \Rightarrow x \in \eta_{\alpha} \Rightarrow x^{u}=\{x\}^{l u} \subseteq$ $\left(\eta_{\alpha}\right)^{l u} \subseteq \eta_{\alpha}$. Thus $x \leq y \Rightarrow y \in x^{u} \Rightarrow y \in \eta_{\alpha}$ and hence $\eta(x)=\alpha \leq \eta(y)$. This proves the result.

Theorem 3.5: Let $(Q, \leq)$ be a lattice. Then an $L$-fuzzy subset $\eta$ of $Q$ is an $L$ - fuzzy closed filter in the poset $Q$ if and only if an $L$-fuzzy filter in the lattice $Q$.

Proof: Let $\eta$ be an $L$-fuzzy filter in the poset $Q$ and $a, b \in$ $Q$. Then $\eta(1)=1$ and since $S=\{a, b\} \subseteq Q$ and $a \wedge b \in S^{l u}$, 
we have $\eta(a \wedge b) \geq \inf \{\eta(x): x \in S\}=\eta(a) \wedge \eta(b)$. Again since $\eta$ is iso-tone, we have $\eta(a \wedge b) \leq \eta(a)$ and $\eta(a \wedge b) \leq$ $\eta(b)$ and hence we have $\eta(a) \wedge \eta(b) \leq \eta(a \wedge b)$. Therefore $\eta(a \wedge b)=\eta(a) \wedge \eta(b)$ and hence $\eta$ is an $L$-fuzzy filter in the lattice $Q$. Conversely suppose $\mu$ be an $L$-fuzzy filter in the lattice $Q$. Then $\eta(1)=1$ and $\eta(a \wedge b)=\eta(a) \wedge \mu(b) \forall a, b \in$ $Q$. Let $S \subseteq Q$ and $x \in(S)^{l u}$. Then $x$ is an upper bound of $(S)^{l}$. Since $\inf S \in(A)^{l}$, we have $x \geq \inf S$ and hence we have $\eta(x) \geq \eta(\inf S)=\inf \{\eta(a): a \in S\}$. Therefore $\eta$ is an $L$-fuzzy closed filter in the poset $Q$. This proves the result.

Lemma 3.6: The intersection of any family of $L$-fuzzy closed filters is an $L$-fuzzy closed filter.

Theorem 3.7: Let $[S)_{C}$ be a closed filter generated by a subset $S$ of $Q$ and $\chi_{S}$ be its characteristic functions. Then the $\left[\chi_{S}\right)=\chi_{[S)_{C}}$.

Proof: Since $[S)_{C}$ is a closed filter of $Q$ containing $S$, by Lemma 3.2, we have $\chi_{[S)_{C}}$ is a fuzzy closed filter. Again since $S \subseteq[S)_{C}$, clearly we have $\chi_{S} \subseteq \chi_{[S)_{C}}$. Now, we show that it is the smallest $L$ - fuzzy closed filter containing $\chi_{S}$. Let $\eta$ be an $L$-fuzzy closed filter such that $\chi_{S} \subseteq \eta$. Then $\eta(a)=1$ for all $a \in S$. Now we claim $\chi_{[S)_{C}} \subseteq \eta$. Let $x \in Q$. If $x \notin[S)_{C}$, then $\chi_{[S)}(x)=0 \leq \eta(x)$. If $x \in[S)_{C}$, then $x \in T^{l u}$ for some subset $T$ of $S$ and hence $\eta(x) \geq \inf \{\eta(b): b \in T\}=1=\chi_{[S)_{C}}(x)$. Hence in either cases, $\chi_{[S)_{C}}(x) \leq \eta(x)$ for all $x \in Q$ and hence $\chi_{[S)_{C}} \subseteq \eta$. This proves the theorem.

In the following theorem we characterize a fuzzy closed filter generated by a fuzzy subset of $Q$ in terms of its level closed filters.

Theorem 3.8: Let $\eta \in L^{Q}$. Then the $L$-fuzzy subset $\hat{\eta}$ of $Q$ defined by $\hat{\eta}(x)=\sup \left\{\alpha \in L: x \in\left[\eta_{\alpha}\right)_{C}\right\}$ for all $x \in Q$ is a fuzzy closed filter of $Q$ generated by $\eta$, where $\left[\mu_{\alpha}\right)_{C}$ is a closed filter generated by $\eta_{\alpha}$.

Proof: Now we show $\hat{\eta}$ is the smallest fuzzy closed filter containing $\eta$. Let $x \in Q$ and put $\eta(x)=\beta$. Then $x \in \eta_{\beta} \subseteq$ $\left[\eta_{\beta}\right)_{C} \Rightarrow \beta \in\left\{\alpha \in L: x \in\left[\eta_{\alpha}\right)_{C}\right\}$. Thus $\eta(x)=\beta \leq \sup \{\alpha \in$ $\left.L: x \in\left[\eta_{\alpha}\right)_{C}\right\}=\hat{\eta}(x)$ and hence $\eta \subseteq \hat{\mu}$. Again since $\{1\}=$ $Q^{u} \subseteq\left[\eta_{\alpha}\right)_{C}$ for all $\alpha \in L$, clearly we have $\hat{\eta}(1)=1$. Let $S$ be any subset of $Q$ and $x \in S^{l u}$. Now $\inf \{\hat{\eta}(a): a \in S\}=$ $\inf \left\{\sup \left\{\alpha_{a}: a \in\left[\eta_{\alpha_{a}}\right)_{C}\right\}: a \in S\right\}=\sup \left\{\inf \left\{\alpha_{a}: a \in S\right\}: a \in\right.$ $\left.\left[\eta_{\alpha_{a}}\right)_{C}\right\}$. Put $\lambda=\inf \left\{\alpha_{a}: a \in S\right\}$. Then $\lambda \leq \alpha_{a}$ for all $a \in S$ and hence $\left[\eta_{\alpha_{a}}\right)_{C} \subseteq\left[\eta_{\lambda}\right)_{C} \forall a \in S$. Therefore $S \subseteq\left[\eta_{\lambda}\right)_{C}$ and hence $x \in S^{l u} \subseteq\left[\eta_{\lambda}\right)^{l u} \subseteq\left[\eta_{\lambda}\right)$. So

$$
\begin{aligned}
\inf \{\hat{\eta}(a): a \in S\} & =\sup \left\{\inf \left\{\alpha_{a}: a \in S\right\}: a \in\left[\eta_{\alpha_{a}}\right)\right\} \\
& \leq \sup \left\{\lambda \in L: x \in\left[\eta_{\lambda}\right)\right\} \\
& =\hat{\eta}(x)
\end{aligned}
$$

Therefore $\hat{\eta}$ is an $L$-fuzzy closed filter. Again let $\theta$ be any $L$-fuzzy closed filter of $Q$ such that $\eta \subseteq \theta$. Then $\eta_{\alpha} \subseteq \theta_{\alpha}$ and $\theta_{\alpha}$ is a closed filter for all $\alpha \in L$ and hence $\left[\eta_{\alpha}\right) \subseteq\left[\theta_{\alpha}\right)=\theta_{\alpha}$. Thus for any $x \in Q, \hat{\eta}(x)=\sup \left\{\alpha \in L: x \in\left[\eta_{\alpha}\right)\right\} \leq \sup \{\alpha \in$ $\left.L: x \in \theta_{\alpha}\right\}=\theta(x)$ and hence $\hat{\eta} \subseteq \theta$. This proves that $\hat{\eta}=[\eta)$.

In the following, we give an algebraic characterization of $L$-fuzzy Closed filter generated by fuzzy subset of $Q$.
Theorem 3.9: Let $\eta \in L^{Q}$. Then the fuzzy subset $\bar{\eta}$ defined by

$$
\bar{\eta}(x)= \begin{cases}1 & \text { if } x=1 \\ \sup \left\{\inf _{a \in S} \eta(a): x \in S^{l u}, S \subseteq Q\right\} & \text { if } x \neq 1\end{cases}
$$

is a fuzzy closed filter of $Q$ generated by $\eta$.

Proof: It is enough to show that $\bar{\eta}=\hat{\eta}$ where $\hat{\eta}$ is an $L$-fuzzy subset given in the above theorem. Let $x \in Q$. If $x=$ 1 , then $\bar{\eta}(x)=1=\hat{\eta}(x)$. Let $x \neq 0$. Put $A_{x}=\left\{\inf _{a \in S} \eta(a)\right.$ : $S \subseteq Q$ and $\left.x \in S^{l u}\right\}$ and $B_{x}=\left\{\alpha: x \in\left[\eta_{\alpha}\right)_{C}\right\}$. Now we show $\sup A_{x}=\sup B_{x}$. Let $\alpha \in A_{x}$. Then $\alpha=\inf _{a \in A} \eta(a)$ for some subset $S$ of $Q$ such that $x \in S^{l u}$. This implies that $\alpha \leq \eta(a)$ for all $a \in S$ and hence $S \subseteq \eta_{\alpha} \subseteq\left[\eta_{\alpha}\right)$. Thus $S^{l u} \subseteq\left(\eta_{\alpha}\right]^{l u} \subseteq$ $\left[\eta_{\alpha}\right)$ and hence $x \in\left[\eta_{\alpha}\right)$. Therefore $\alpha \in B_{x}$. Thus $A_{x} \subseteq B_{x}$ and hence $\sup A_{x} \leq \sup B_{x}$. Again let $\alpha \in B_{x}$.Then $x \in\left[\eta_{\alpha}\right)$. Since $\left[\mu_{\alpha}\right)_{C}=\bigcup\left\{S^{l u}: S \subseteq \eta_{\alpha}\right\}$, we have $x \in S^{l u}$ for some subset $S$ of $\eta_{\alpha}$. This implies $\eta(a) \geq \alpha$ for all $a \in S$ and hence $\inf \{\eta(a): a \in S\} \geq \alpha$. Thus $\beta=\inf \{\eta(a): a \in S\} \in A_{x}$. Thus for each $\alpha \in B_{x}$ we get $\beta \in A_{x}$ such that $\alpha \leq \beta$ and hence $\sup A_{x} \geq \sup B_{x}$. Therefore $\sup A_{x}=\sup B_{x}$ and hence $\bar{\eta}=\hat{\eta}$.

The above result yields the following.

Theorem 3.10: Let $\mathscr{F} \mathscr{C} \mathscr{F}(Q)$ be the set of all $L$-fuzzy closed filters of $Q$. Then $(\mathscr{F} \mathscr{C} \mathscr{F}(Q), \subseteq)$ forms a complete lattice with respect to the point wise ordering " $\subseteq$ ", in which the supremum $\sup _{i \in \Delta} \mu_{i}$ and the inifimum $\inf _{i \in \Delta} \eta_{i}$ of any family $\left\{\eta_{i}: i \in \Delta\right\}$ in $\mathscr{F} \mathscr{C} \mathscr{F}(Q)$ are given by:

$\sup _{i \in \Delta} \eta_{i}=\overline{\bigcup_{i \in \Delta}\left\{\eta_{i}\right\}}$ and $\inf _{i \in \Delta} \eta_{i}=\bigcap_{i \in \Delta} \eta_{i}$.

Corollary 3.11: For any $L$-fuzzy closed filters $\eta$ and $v$ of $Q$, the supremum $\eta \vee v$ and the infimum $\eta \wedge v$ of $\eta$ and $v$ in $\mathscr{F} \mathscr{C} \mathscr{F}(Q)$ respectively are:

$\eta \vee v=\overline{\eta \cup v}$ and $\eta \wedge v=\eta \cap v$.

Now we introduce the fuzzy version of a filter (dual ideal) of a poset introduced by O. Frink [4].

Definition 3.12: An $L$-fuzzy subset $\eta$ of $Q$ is an $L$-fuzzy Frink filter if it satisfies the following conditions:

1) $\eta(1)=1$ and

2) for any finite subset $F$ of $Q, \eta(x) \geq \inf \{\eta(a): a \in F\}$ $\forall x \in F^{l u}$

Lemma 3.13: Let $\eta \in L^{Q}$. Then $\eta$ is an $L$-fuzzy Frink filter of $Q$ if and only if $\eta_{\alpha}$ is a Frink filter of $Q$ for all $\alpha \in L$.

Lemma 3.14: Let $\eta$ be fuzzy Frink filter of a poset $Q$. Then $\eta$ is iso-tone, in the sense that $\eta(x) \leq \eta(y)$ whenever $x \leq y$.

Corollary 3.15: A subset $S$ of $Q$ is a Frink filter of $Q$ if and only if its characteristic map $\chi_{S}$ is an $L$-fuzzy Frink filter of $Q$.

Theorem 3.16: Let $(Q, \leq)$ be a lattice and $\eta \in L^{Q}$. Then $\eta$ is an $L$ - fuzzy Frink filter in the poset $Q$ if and only it an $L$-fuzzy filter in the lattice $Q$.

Lemma 3.17: The intersection of any family of $L$-fuzzy Frink-filters is an $L$-fuzzy Frink filter.

Theorem 3.18: Let $[S)_{F}$ be a Frink-filter generated by subset $S$ of $Q$ and $\chi_{S}$ be its characteristic functions. Then $\left[\chi_{S}\right)=\chi_{[S)_{F}}$. In the following theorems, we give characterizations of $L$ Fuzzy Frink filters generated by fuzzy subset of $Q$.

Theorem 3.19: Let $\eta \in L^{Q}$. Define a fuzzy subset $\hat{\eta}$ of $Q$ by $\hat{\eta}(x)=\sup \left\{\alpha \in L: x \in\left[\eta_{\alpha}\right)_{F}\right\}$ for all $x \in Q$ where $\left[\eta_{\alpha}\right)_{F}$ a 
Frink filter generated by $\eta_{\alpha}$, where $\left[\eta_{\alpha}\right)_{F}$ is a Frink filter generated by $\eta_{\alpha}$. Then $\hat{\eta}$ is an $L$-fuzzy Frink filter of $Q$ generated by $\eta$.

In the following, we give an algebraic characterization of $L$ fuzzy Frink filters generated by fuzzy subset of $Q$.

Theorem 3.20: Let $\eta$ be a fuzzy subset of $Q$. Then the fuzzy subset $\vec{\eta}$ defined by

$$
\vec{\eta}(x)= \begin{cases}1 & \text { if } x=1 \\ \sup \left\{\inf _{a \in F} \eta(a): F \subset \subset Q, x \in F^{l u}\right\} & \text { if } x \neq 1\end{cases}
$$

is a Frink fuzzy filter of $Q$ generated by $\eta$.

Theorem 3.21: Let $\mathscr{F} \mathscr{F} \mathscr{F}(Q)$ be the of all $L$-fuzzy Frink filter of $Q$. Then $(\mathscr{F} \mathscr{F} \mathscr{F}(Q), \subseteq)$ forms a complete lattice with respect to point wise ordering " $\subseteq$ ", in which the supremum and the infimum of any family $\left\{\eta_{i}: i \in \Delta\right\}$ in $\mathscr{F} \mathscr{F} \mathscr{F}(Q)$ respectively are: $\sup _{i \in \Delta} \eta_{i}=\widehat{\bigcup_{i \in \Delta}\left\{\eta_{i}\right\}}$ and $\inf _{i \in \Delta} \eta_{i}=\bigcap_{i \in \Delta} \eta_{i}$.

Corollary 3.22: For any $L$-fuzzy Frink ideals $\eta$ and $v$ of $Q$ in the supremum $\eta \vee v$ and the infimum $\eta \wedge v$ of $\eta$ and $v$ in $\mathscr{F} \mathscr{F} \mathscr{F}(Q)$ respectively are: $\eta \vee v=\overrightarrow{\eta \cup v}$ and $\eta \wedge v=\eta \cap v$. Now we introduce the fuzzy version of semi-filters and $\mathrm{V}$ filters of a poset introduced by P.V. Venkatanarasimhan [5] and [6].

Definition 3.23: $\eta$ in $L^{Q}$ is said to be an $L$-fuzzy semi-filter or $L$-fuzzy order filter if $\eta(x) \leq \eta(y)$ whenever $x \leq y$ in $Q$.

Definition 3.24: $\eta$ in $L^{Q}$ is said to be an $L$ - fuzzy $V$-filter if it satisfies the following conditions:

1) for any $x, y \in Q \eta(x) \leq \eta(y)$ whenever $x \leq y$ and

2) for any non-empty finite subset $B$ of $Q$, if $\inf B$ exists then $\eta(\inf B) \geq \inf \{\eta(b): b \in B\}$.

Theorem 3.25: Every $L$-fuzzy Frink filter is an $L$-fuzzy $V$ filter.

Proof: Let $\eta$ be an $L$-fuzzy Frink filter and let $x, y \in Q$ such that $x \leq y$. Put $\eta(x)=\alpha$. Since $\eta$ is an $L$-fuzzy Frink filter, $\eta_{\alpha}$ is a Frink filter of $Q$. Now $\eta(x)=\alpha \Rightarrow x \in \eta_{\alpha} \Rightarrow$ $\{x\}^{l u} \subseteq \eta_{\alpha}$. Now $x \leq y \Rightarrow y \in x^{u}=x^{l u} \subseteq \eta_{\alpha} \Rightarrow \eta(x)=\alpha \leq$ $\eta(y)$. Again let $B$ be any nonempty subset of $Q$ such that inf $B$ exists in $Q$. Then $\inf B \in B^{l u}$ and hence $\eta(\inf B) \geq \inf \{\eta(a)$ : $a \in B\}$. Therefore $\eta$ is an $L$-fuzzy $V$-filter.

Now we introduce the fuzzy version filters of a poset introduced by Halaš [7] which seems to be a suitable generalization of the usual concept of $L$-fuzzy filter of a lattice.

Definition 3.26: $\eta \in L^{Q}$ is called an $L$ - fuzzy filter in the sense of Halaš if it fulfills the followings:

1) $\eta(1)=1$ and

2) for any $a, b \in Q, \eta(x) \geq \eta(a) \wedge \eta(b)$ for all $x \in(a, b)^{l u}$

In the rest of this paper, an $L$-fuzzy filter of a poset will mean an $L$-fuzzy filter in the sense of Halaš.

Lemma 3.27: $\eta \in L^{Q}$ is an $L$-fuzzy filter of $Q$ if and only if $\eta_{\alpha}$ is a filter of $Q$ in the sense of Halaš for all $\alpha \in L$.

Corollary 3.28: A subset $S$ of $Q$ is a filter of $Q$ in the sense of Halaš if and only if its characteristic map $\chi_{S}$ is an $L$-fuzzy filter of $Q$.

Lemma 3.29: If $\eta$ is an $L$-fuzzy filter of $Q$, then the following assertions hold:

1) for any $x, y \in Q \eta(x) \leq \eta(y)$ whenever $x \leq y$.

2) for any $x, y \in Q, \eta(x \wedge y) \geq \mu(x) \wedge \eta(y)$ whenever $x \wedge y$ exists.
Theorem 3.30: Let $(Q, \leq)$ be a lattice. Then an $L$-fuzzy subset $\eta$ of $Q$ is an $L$-fuzzy filter in the poset $Q$ if and only if an $L$-fuzzy filter is in the lattice $Q$.

Theorem 3.31: Let $[S)_{H}$ be a filter generated by subset $S$ of $Q$ in the sense of Halaš and $\chi_{S}$ be its characteristic functions. Then $\left[\chi_{S}\right)=\chi_{[S)_{H}}$.

Lemma 3.32: The intersection of any family of $L$-fuzzy filters is an $L$ - fuzzy filter.

Now we give characterization of an $L$ - fuzzy filter generated by a fuzzy subset of a poset $Q$.

Definition 3.33: Let $\eta$ be a fuzzy subset of $Q$ and $\mathscr{N}$ be a set of positive integers. Define fuzzy subsets of $Q$ inductively as follows: $B_{1}^{\eta}(x)=\sup \left\{\eta(a) \wedge \eta(b): x \in(a, b)^{l u}\right\}$ and $B_{n}^{\eta}(x)=$ $\sup \left\{B_{n-1}^{\eta}(a) \wedge B_{n-1}^{\eta}(b): x \in(a, b)^{l u}\right\}$ for each $n \geq 2$ and $a, b \in$ $Q$.

Theorem 3.34: The set $\left\{B_{n}^{\eta}: n \in \mathscr{N}\right\}$ forms a chain and the fuzzy subset $\hat{\eta}$ defined by $\hat{\eta}(x)=\sup \left\{B_{n}^{\eta}(x): n \in \mathscr{N}\right\}$ is a fuzzy filter generated by $\eta$.

Proof: Let $x \in Q$ and $n \in \mathscr{N}$. Then

$$
\begin{aligned}
B_{n+1}^{\eta}(x) & =\sup \left\{B_{n}^{\eta}(a) \wedge B_{n}^{\eta}(b): x \in(a, b)^{l u}\right\} \\
& \geq B_{n}^{\eta}(x) \wedge B_{n}^{\eta}(x)\left(\text { since } x \in x^{u}=(x, x)^{l u}\right) \\
& =B_{n}^{\eta}(x) \forall x \in Q .
\end{aligned}
$$

Therefore $B_{n}^{\eta} \subseteq B_{n+1}^{\eta}$ for each $n \in \mathscr{N}$ and hence $\left\{B_{n}^{\eta}: n \in\right.$ $\mathscr{N}\}$ is a chain. Now we show $\hat{\eta}$ is the smallest fuzzy filter containing $\eta$.

$$
\begin{aligned}
\text { Since } \hat{\eta}(x) & =\sup \left\{B_{n}^{\eta}(x): n \in \mathscr{N}\right\} \\
& \geq B_{1}^{\eta}(x) \\
& =\sup \left\{\eta(a) \wedge \eta(b): x \in(a, b)^{l u}\right\} \\
& \geq \eta(x) \wedge \eta(x) \quad\left(\text { since } x \in(x, x)^{l u}\right) \\
& =\eta(x) \forall x \in Q .
\end{aligned}
$$

Therefore $\eta \subseteq \hat{\eta}$. Let $a, b \in L$ and $x \in(a, b)^{l u}$.

$$
\begin{aligned}
\text { Now } \hat{\eta}(x)= & \sup \left\{B_{n}^{\eta}(x): n \in \mathscr{N}\right\} \\
\geq & B_{n}^{\eta}(x) \text { for all } n \in \mathscr{N} \\
= & \sup \left\{B_{n-1}^{\eta}(y) \wedge B_{n-1}^{\eta}(z): x \in(y, z)^{l u}\right\} \\
& \quad \text { for all } n \geq 2 . \\
\geq & B_{n-1}^{\eta}(a) \wedge B_{n-1}^{\eta}(b) \forall n \geq 2 \\
& \left(\text { since } x \in(a, b)^{u l}\right) \\
= & B_{m}^{\eta}(a) \wedge B_{m}^{\eta}(b) \forall m \in \mathscr{N}
\end{aligned}
$$

$$
\begin{aligned}
\text { Thus } \hat{\eta}(x) \geq & \sup \left\{B_{m}^{\eta}(a) \wedge B_{m}^{\eta}(b): m \in \mathscr{N}\right\} \\
= & \sup \left\{B_{m}^{\eta}(a): m \in \mathscr{N}\right\} \wedge \\
& \sup \left\{B_{m}^{\eta}(b): m \in \mathscr{N}\right\} \\
= & \hat{\eta}(a) \wedge \hat{\eta}(b) .
\end{aligned}
$$

Therefore $\hat{\eta}$ is a fuzzy filter. Again let $\theta$ be any $L$-fuzzy filter of $Q$ such that $\eta \subseteq \theta$. Now let $a, b \in Q$ and $x \in$ $(a, b)^{l u}$. Then $\theta(x) \geq \theta(a) \wedge \theta(b) \geq \eta(a) \wedge \eta(b)$. This implies $\theta(x) \geq \sup \left\{\eta(a) \wedge \eta(b): x \in(a, b)^{u l}\right\}=B_{1}^{\eta}(x)$. Therefore $\theta(x) \geq B_{1}^{\eta}(x)$ for all $x \in(a, b)^{l u}$. Again for any $x \in(a, b)^{l u}$ we have $\theta(x) \geq \theta(a) \wedge \theta(b) \geq B_{1}^{\eta}(a) \wedge B_{1}^{\eta}(b)$. This implies $\theta(x) \geq \sup \left\{B_{1}^{\eta}(a) \wedge B_{1}^{\eta}(b): x \in(a, b)^{l u}\right\}=B_{2}^{\eta}(x)$. Thus by 
induction we have $\theta(x) \geq B_{n}^{\eta}(x) \forall n \in \mathscr{N}$ and $\forall x \in(a, b)^{l u}$. Thus for any $x \in Q$, we have

$$
\begin{aligned}
\hat{\eta}(x)= & \sup \left\{B_{n}^{\eta}(x): n \in \mathscr{N}\right\} \\
= & \sup \left\{B_{n-1}^{\eta}(a) \wedge B_{n-1}^{\eta}(b): n \in \mathscr{N}, x \in(a, b)^{l u}\right\} \\
\leq & \sup \left\{\theta(a) \wedge \theta(b): x \in(a, b)^{l u}\right\} \\
& \left(\operatorname{since}, a, b \in(a, b)^{l u} .\right) \\
\leq & \theta(x)
\end{aligned}
$$

Therefore $\theta \supseteq \hat{\eta}$. This proves the theorem.

The above result yields the following.

Theorem 3.35: Let $\mathscr{F} \mathscr{F}(Q)$ be the set of all $L$-fuzzy filter of $Q$. Then $(\mathscr{F} \mathscr{F}(Q), \subseteq)$ forms a complete lattice with respect to the point wise ordering " $\subseteq$ ", in which the supremum and the infimum of any family $\left\{\eta_{i}: i \in \Delta\right\}$ in $\mathscr{F} \mathscr{F}(Q)$ respectively are: $\left(\sup _{i \in \Delta} \eta_{i}\right)(x)=\sup \left\{B_{n}^{\bigcup} \bigcup_{i \in \Delta} \eta_{i}(x): n \in \mathscr{N}\right\}$ and $\left(\inf _{i \in \Delta} \eta_{i}\right)(x)=$ $\left(\bigcap_{i \in \Delta} \eta_{i}\right)(x)$ for any $x \in Q$.

Corollary 3.36: For any $L$-fuzzy filter $\eta$ and $v$ of $Q$, the supremum $\eta \vee v$ and the infimum $\eta \wedge v$ of $\eta$ and $v$ in $\mathscr{F} \mathscr{F}(Q)$ respectively are: $(\eta \vee v)(x)=\sup \left\{B_{n}^{\eta \cup v}(x): n \in \mathscr{N}\right\}$ and $(\eta \wedge$ $v)(x)=(\eta \cap v)(x)$ for any $x \in Q$.

Theorem 3.37: The following implications hold, where all of them are not equivalent:

1) $L$-fuzzy closed filter $\Longrightarrow L$-fuzzy Frink filter $\Longrightarrow L$-fuzzy $V$-filter $\Longrightarrow L$-fuzzy semi-filter.

2) $L$ - fuzzy closed filter $\Longrightarrow L$-fuzzy Frink filter $\Longrightarrow L$ fuzzy filter $\Longrightarrow L$ - fuzzy semi-filter.

The following examples show that the converse of the above implications do not hold in general.

Example 3.38: Consider the Poset $([0,1], \leq)$ with the usual ordering. Define a fuzzy subset $\eta:[0,1] \longrightarrow[0,1]$ by

$$
\eta(x)= \begin{cases}1 & \text { if } x \in\left(\frac{1}{2}, 1\right] \\ 0 & \text { if } x \in\left[0, \frac{1}{2}\right]\end{cases}
$$

Then $\eta$ is an $L$ - fuzzy Frink filter but not an $L$ - fuzzy closed filter.

Example 3.39: Consider the poset $(Q, \leq)$ depicted in the figure below. Define a fuzzy subset $v: Q \longrightarrow[0,1]$ by $v(1)=$ $v\left(a^{\prime}\right)=1, v(a)=v(b)=v(c)=v(d)=v(0)=0.2, v\left(b^{\prime}\right)=$ $0.6, v\left(c^{\prime}\right)=0.5$ and $v\left(d^{\prime}\right)=0.7$. Then $v$ is an $L$-fuzzy filter

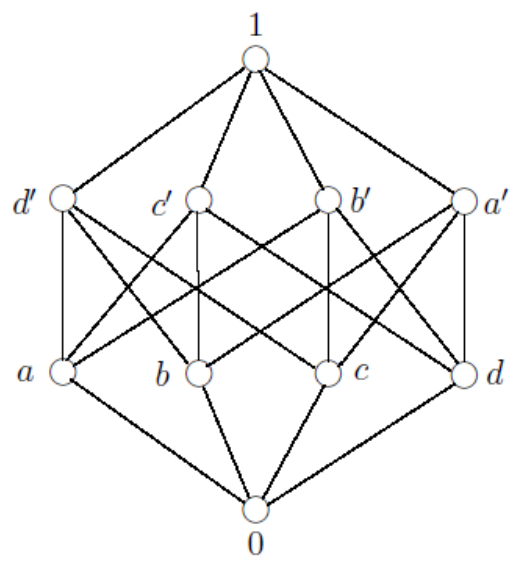

Fig. 1. A Poset. but not an $L$ - fuzzy Frink-filter.

Example 3.40: Consider the poset $(Q, \leq)$ depicted in the figure below. Define a fuzzy subset $\theta: Q \longrightarrow[0,1]$ by $\theta(U)=$ 1, $\theta(L)=\theta(M)=0.8$ and $\theta(N)=0.6$. Then $\theta$ is an $L$-fuzzy

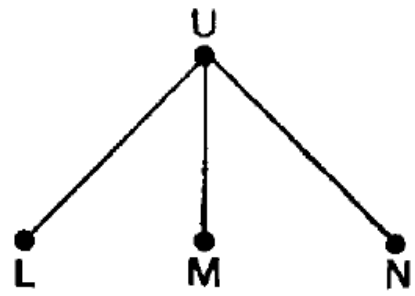

Fig. 2. A Poset.

V-filter but not an $L$ - fuzzy Frink-filter.

Example 3.41: Consider the poset $(Q, \leq)$ depicted in the figure below. Define a fuzzy subset $\sigma: Q \longrightarrow[0,1]$ by $\sigma(1)=$ $1, \sigma(a)=0.8, \sigma(b)=0.9$ and $\sigma(0)=0.2$.

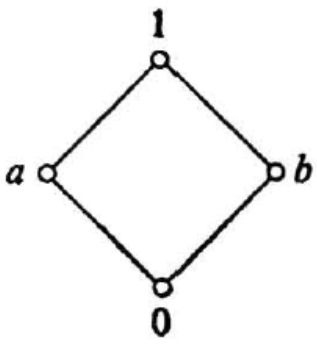

Fig. 3. A Poset.

Then $\sigma$ is an $L$-fuzzy semi-filter but not an $L$-fuzzy filter.

Theorem 3.42: Let $x \in Q$ and $\alpha \in L$. Define an $L$ - fuzzy subset $\alpha^{x}$ of $Q$ by

$$
\alpha^{x}(y)= \begin{cases}1 & \text { if } y \in[x) \\ \alpha & \text { if } y \notin[x)\end{cases}
$$

for all $y \in Q$. Then $\alpha^{x}$ is an $L$-fuzzy filter of $Q$.

Proof: By the definition of $\alpha^{x}$, we clearly have $\alpha_{x}(1)=1$. Let $a, b \in Q$ and $y \in(a, b)^{l u}$. Now if $a, b \in[x)$, then we have $(a, b)^{l u} \subseteq[x)$ and $\alpha^{x}(a)=\alpha^{x}(b)=1$. Thus $\alpha^{x}(y)=1=1 \wedge 1=$ $\alpha^{x}(a) \wedge \alpha^{x}(b)$. Again if $a \notin[x)$ or $b \notin[x)$, we have $\alpha^{x}(a) \wedge$ $\alpha^{x}(b)=\alpha$ and hence $\alpha^{x}(y) \geq \alpha=\alpha^{x}(a) \wedge \alpha^{x}(b)$. Therefore in either cases we have $\alpha^{x}(y) \geq \alpha^{x}(a) \wedge \alpha^{x}(b)$ for all $y \in(a, b)^{l u}$ and hence $\alpha^{x}$ is an $L$-fuzzy filter.

Definition 3.43: The $L$-fuzzy filter $\alpha^{x}$ defined above is called the $\alpha$-level principal fuzzy filter corresponding to $x$.

Definition 3.44: An $L$-fuzzy filter $\mu$ of a poset $Q$ is called an $l$ - $L$-fuzzy filter if for any $a, b \in Q$, there exists $x \in(a, b)^{l}$ such that $\mu(x)=\mu(a) \wedge \mu(b)$.

Lemma 3.45: An $L$-fuzzy filter $\mu$ of $Q$ is an $l$ - $L$-fuzzy filter of $Q$ if and only if $\mu_{\alpha}$ is an $l$-filter of $Q$ for all $\alpha \in L$.

Proof: Suppose $\mu$ is an $l$ - $L$-fuzzy filter and $\alpha \in L$. Since $\mu$ is an $L$ - fuzzy filter, $\mu_{\alpha}$ is a filter of $Q$. Let $a, b \in \mu_{\alpha}$. Then $\mu(a) \geq \alpha$ and $\mu(b) \geq \alpha$ and hence $\mu(a) \wedge \mu(b) \geq \alpha$. Also since $\mu$ is an $l$ - $L$ - fuzzy filter there exists $x \in(a, b)^{l}$ such that $\mu(x)=$ $\mu(a) \wedge \mu(b)$ and hence $\mu(x) \geq \alpha$. Therefore $x \in \mu_{\alpha} \cap(a, b)^{l}$ 
and hence $\mu_{\alpha} \cap(a, b)^{l} \neq \emptyset$. Therefore $\mu_{\alpha}$ is an $l$-filter of a poset $Q$. Conversely suppose $\mu_{\alpha}$ is an $l$-filter of a poset $Q$ for all $\alpha \in L$. Then $\mu$ is an $L$-fuzzy filter. Let $a, b \in Q$ and put $\alpha=$ $\mu(a) \wedge \mu(b)$. Then $\mu_{\alpha} \cap(a, b)^{l} \neq \emptyset$. Let $x \in \mu_{\alpha} \cap(a, b)^{l}$. Then $x \in \mu_{\alpha}$ and $x \in(a, b)^{l}$. This implies $\mu(x) \geq \alpha=\mu(a) \wedge \mu(b)$ and $x \leq a, x \leq b$. Since $\mu$ is iso-tone we have $\mu(x) \leq \mu(a)$ and $\mu(x) \leq \mu(b)$ and hence $\mu(x) \leq \mu(a) \wedge \mu(b)$. Therefore there exists $x \in(a, b)^{l}$ such that $\mu(x)=\mu(a) \wedge \mu(b)$ and hence $\mu$ is an $l$ - $L$-fuzzy filter.

Corollary 3.46: Let $(Q, \leq)$ be a poset with 0 and let $x \in Q$ and $\alpha \in L$. Then the $\alpha$-level principal fuzzy filter corresponding to $x$ is an $l$ - $L$-fuzzy filter.

Remark 3.47: Every $L$-fuzzy filter is not an $l$ - $L$-fuzzy filter. For example consider the poset $(Q, \leq)$ depicted in the figure below and define a fuzzy subset $\mu: Q \longrightarrow[0,1]$ by $\mu(1)=1$, $\mu(c)=\mu(d)=0.9, \mu(a)=\mu(b)=\mu(0)=0.7$. Then $\mu$ is an $L$-fuzzy filter but not an $l$ - $L$-fuzzy filter.

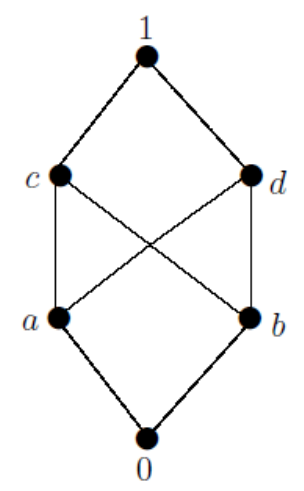

Fig. 4. A Poset.

Theorem 3.48: Every $l$ - $L$-fuzzy filter is an $L$ - fuzzy Frink filter.

Proof: Suppose $\eta$ is an $l$ - $L$-fuzzy filter. Let $F$ be a finite subset of $Q$. Then there exists $y \in F^{l}$ such that $\eta(y)=$ $\inf \{\eta(a): a \in F\}$.

$$
\text { Again } \begin{aligned}
x \in F^{l u} & \Rightarrow s \leq x \forall s \in F^{l} \\
& \Rightarrow y \leq x\left(\text { since } y \in F^{l}\right) \\
& \Rightarrow \eta(x) \geq \eta(y)=\inf \{\eta(a): a \in F\} \\
& \Rightarrow \eta(x) \geq \inf \{\eta(a): a \in F\}
\end{aligned}
$$

Therefore $\eta$ is an $L$-fuzzy Frink filter.

Theorem 3.49: Let $\eta$ and $\theta$ be $l$ - $L$-fuzzy filters of $Q$. Then the supremum $\eta \vee \theta$ of $\eta$ and $\theta$ in $\mathscr{F} \mathscr{F}(Q)$ is given by: $(\eta \vee \theta)(x)=\sup \left\{\eta(a) \wedge \theta(b): x \in(a, b)^{l u}\right\}$ for all $x \in Q$.

Proof: Let $\sigma$ be an $L$-fuzzy subset of $Q$ defined by $\sigma(x)=$ $\sup \left\{\eta(a) \wedge \theta(b): x \in(a, b)^{l u}\right\} \forall x \in Q$. Now we claim $\sigma$ is the smallest $L$-fuzzy filter of $Q$ containing $\eta \cup \theta$. Let $x \in Q$.

$$
\begin{aligned}
\text { Now } \sigma(x) & =\sup \left\{\eta(a) \wedge \theta(b): x \in(a, b)^{l u}\right\} \\
& \geq \eta(x) \wedge \theta(1),\left(\text { since } x \in(x, 1)^{l u}\right) \\
& =\eta(x) \wedge 1=\eta(x)
\end{aligned}
$$

and hence $\sigma \supseteq \eta$. Similarly we can show $\sigma \supseteq \theta$ and hence $\sigma \supseteq \eta \cup \theta$
Let $a, b \in Q$ and $x \in(a, b)^{l u}$. Now

$$
\begin{aligned}
\sigma(a) \wedge \sigma(b)= & \sup \left\{\eta(c) \wedge \theta(d): a \in(c, d)^{l u}\right\} \wedge \\
& \sup \left\{\eta(e) \wedge \theta(f): b \in(e, f)^{l u}\right\} \\
= & \sup \{\eta(c) \wedge \theta(d) \wedge \eta(e) \wedge \theta(f): \\
& \left.a \in(c, d)^{l u}, b \in(e, f)^{l u}\right\} \\
\leq & \sup \{\eta(c) \wedge \eta(e) \wedge \theta(d) \wedge \theta(f): \\
& \left.a, b \in(c, d, e, f)^{l u}\right\}
\end{aligned}
$$

Again since $\eta$ and $\theta$ are $l$-L-fuzzy filters, for each $c, e$ and $d, f$ there are $r \in(c, e)^{l}$ and $s \in(d, f)^{l}$ such that $\eta(r)=\eta(c) \wedge \eta(e)$ and $\theta(s)=\theta(d) \wedge \theta(f)$. Now

$$
\begin{aligned}
r \in(c, e)^{l} \text { and } s \in(d, f)^{l} & \Rightarrow\{c, d, e, f\}^{l u} \subseteq\{s, r\}^{l u} \\
& \Rightarrow a, b \in\{s, r\}^{l u} \\
& \Rightarrow(a, b)^{l u} \subseteq\{s, r\}^{l u} \\
& \Rightarrow x \in\{s, r\}^{l u}
\end{aligned}
$$

Thus $\sigma(a) \wedge \sigma(b) \leq \sup \{\eta(c) \wedge \eta(e) \wedge \theta(d) \wedge \theta(f): a, b \in$ $\left.(c, d, e, f)^{l u}\right\} \leq \sup \left\{\eta(r) \wedge \theta(s): x \in(r, s)^{l u}\right\} \leq \sigma(x)$ for all $x \in(a, b)^{l u}$ and hence $\sigma$ is an $L$-fuzzy filter.

Let $\phi$ be any $L$-fuzzy filter of $Q$ such that $\eta \cup \theta \subseteq \phi$. Now for any $x \in Q$, we have

$$
\begin{aligned}
\sigma(x) & =\sup \left\{\eta(a) \wedge \theta(b): x \in(a, b)^{l u}\right\} \\
& \leq \sup \left\{\phi(a) \wedge \phi(b): x \in(a, b)^{l u}\right\} \\
& \leq \phi(x)
\end{aligned}
$$

and hence $\sigma \subseteq \phi$. Therefore $\sigma=(\eta \cup \theta]=\eta \vee \theta$, that is $\sigma$ is the supremum of $\eta$ and $\theta$ in $\mathscr{F} \mathscr{F}(Q)$.

\section{REFERENCES}

[1] B. Alaba, M. Taye, and D. Engidaw, "L-fuzzy ideals of a poset," Ann. Fuzzy Math. Inform., vol. 16, pp. 285-299, 2018.

[2] G. Birkhoff, Lattice theory. American Mathematical Soc., 1940, vol. 25.

[3] M. Stone, "The theory of representation for boolean algebras," Transactions of the American Mathematical Society, vol. 40, no. 1, pp. 37-111, 1936.

[4] O. Frink, "Ideals in partially ordered sets," The American Mathematical Monthly, vol. 61, no. 4, pp. 223-234, 1954.

[5] P. Venkatanarasimhan, "Pseudo-complements in posets," Proceedings of the American Mathematical Society, vol. 28, no. 1, pp. 9-17, 1971

[6] _- "Semi-ideals in posets," Mathematische Annalen, vol. 185, no. 4, pp. 338-348, 1970.

[7] R. Halaš, "Annihilators and ideals in ordered sets," Czechoslovak Mathematical Journal, vol. 45, no. 1, pp. 127-134, 1995.

[8] N. Ajmal and K. Thomas, "Fuzzy lattices," Information sciences, vol. 79, no. 3-4, pp. 271-291, 1994.

[9] B. Koguep, C. Nkuimi, and C. Lele, "On fuzzy prime ideals of lattice," SJPAM, vol. 3, pp. 1-11, 2008

[10] T. Rao, C. Rao, D. Solomon, and D. Abeje, "Fuzzy ideals and filters of lattices," Asian Journal of Current Engineering and Maths, vol. 2, no. $4,2013$.

[11] U. Swamy and D. Raju, "Fuzzy ideals and congruences of lattices," Fuzzy sets and systems, vol. 95, no. 2, pp. 249-253, 1998.

[12] Y. Bo and W. Wangming, "Fuzzy ideals on a distributive lattice," Fuzzy sets and systems, vol. 35, no. 2, pp. 231-240, 1990.

[13] B. Davey and H. Priestley, Introduction to lattices and order. Cambridge university press, 2002

[14] G. Grätzer, General lattice theory. Springer Science \& Business Media, 2002.

[15] R. Halaš and J. Rachŭnek, "Polars and prime ideals in ordered sets," Discuss. Math., Algebra Stoch. Methods, vol. 15, pp. 43-59, 1995.

[16] J. Goguen, "L-fuzzy sets," Journal of mathematical analysis and applications, vol. 18, no. 1, pp. 145-174, 1967. 
[17] L. Zadeh, "Fuzzy sets," Information and control, vol. 8, no. 3, pp. 338353, 1965.

[18] J. Mordeson and D. Malik, Fuzzy commutative algebra. World scientific, 1998. 